\title{
Nonlinear Homogenization Algorithms with Low Computational Cost*
}

\author{
Jun-ichi OKADA $^{* *}$, Takumi WASHIO*** and Toshiaki HISADA ${ }^{\dagger}$ \\ ** Graduate School of Frontier Sciences, University of Tokyo, \\ 5-1-5 Kashiwanoha, Kashiwa, Chiba 277-8563, Japan \\ E-mail: okada @sml.k.u-tokyo.ac.jp \\ ${ }^{* * *}$ Graduate School of Frontier Sciences, University of Tokyo, \\ 5-1-5 Kashiwanoha, Kashiwa, Chiba 277-8563, Japan \\ E-mail: washio@sml.k.u-tokyo.ac.jp \\ 'Graduate School of Frontier Sciences, University of Tokyo, \\ 7-3-1 Hongo, Bunkyo-ku, Tokyo 113-0031, Japan \\ E-mail: hisada@mech.t.u-tokyo.ac.jp
}

\begin{abstract}
An efficient homogenization method for nonlinear problems is introduced. We have already developed a homogenization technique using characteristic deformation mode superposition that avoids prohibitive computational cost. However, in the mode superposition technique, the approximation error created depends on the analysis case. In this paper a new method is proposed, in which the same accuracy as the exact method is preserved by solving the microscopic equilibrium equation, while approximating the tangential matrix of the multi-scale equilibrium equation using the mode superposition method. The performance of the proposed method is examined together with the block LU factorization algorithm, and satisfactory results are obtained.
\end{abstract}

Key words : Homogenization Method, Nonlinear, Finite Element Analysis, Algorithm, Block LU Factorization, Heart

\section{Introduction}

The homogenization method is a mathematical modeling technique for efficiently analyzing inhomogeneous material with a periodic microstructure. To measure the spatial change in the domain, we introduce two scales, that is, a scale for the unit period, and a scale for the whole material. By solving the governing equations of both scales with coupling, we can obtain the macroscopic characteristic as an equivalent homogeneous body and variable distribution from the microstructure. To investigate the effect of intracellular structure on heartbeat, we are developing the necessary finite element method which is calculated with the heart as the macrostructure, and the cardiomyocyte as the microstructure.

Biomaterial is usually modeled by hyperelastic material. However, the myofibril in the cardiomyocyte generates contraction forces and stiffness that is changed by chemical reaction and includes high nonlinearity. In the conventional nonlinear homogenization method ${ }^{(1)(2)}$, it is necessary to calculate microscopic equilibrium and macroscopic tangential homogenization updates at all quadrature points in every Newton-Raphson iteration, resulting in huge computational cost. To reduce this computational cost, various techniques have been devised. These include, for example, the construction of a database with the homogenized properties ${ }^{(3)}$, sensitivity analysis ${ }^{(4)}$, Fast Fourier Transforms ${ }^{(5)}$, and so on. In a previous paper, to circumvent these difficulties, we proposed the basic framework of a homogenization method that reduces computational cost by using characteristic deformation mode superposition ${ }^{(6)}$. This method is applicable to the microstructure, which is composed of nearly incompressible and viscoelastic materials ${ }^{(7)(8)}$. However, depending on the type of analysis, a significant error may be caused by this technique. 
In this paper, to achieve macroscopic equilibrium under the given boundary condition and a microscopic self-equilibrium condition efficiently, we propose two new algorithms. First, we show that the algorithm, which is the residual of each scale, decreases simultaneously using block LU factorization without microscopic convergence in the macroscopic iteration as in the conventional algorithm. In addition, an algorithm which approximates the homogenization tangent stiffness using the mode superposition method is proposed. In this method, the same solution as in the original method is preserved with far less computational cost by solving the microscopic equilibrium equation alternately with the solution of the mode superpositionbased multi-scale equilibrium equation. Finally, the performance of the proposed method is examined and compared with the conventional method and an example of a simplified multiscale heart analysis is presented.

\section{Nomenclature}

\begin{tabular}{|c|c|}
\hline $\mathbf{Y}, \mathbf{y}$ & :position vector around the deformation in the microstructure \\
\hline $\mathbf{X}, \mathbf{x}$ & :position vector around the deformation in the macrostructure \\
\hline $\mathbf{u}$ & :macroscopic displacement vector \\
\hline$\{\mathbf{u}\}$ & :macroscopic structure nodal displacement vector \\
\hline$\left\{\mathbf{u}^{e}\right\}$ & :macroscopic structure nodal displacement vector per element \\
\hline $\mathbf{w}$ & :periodic component of the microscopic displacement vector \\
\hline$\{\mathbf{w}\}$ & $\begin{array}{l}\text { :periodic component of the nodal displacement } \\
\text { vectors of all microstructures }\end{array}$ \\
\hline$\left\{\mathbf{w}^{Q}\right\}$ & $\begin{array}{l}\text { :periodic component of the nodal displacement } \\
\text { vector of a single microstructure }\end{array}$ \\
\hline$\left\{\mathbf{w}^{e}\right\}$ & :periodic component of the nodal displacement vector per element \\
\hline $\mathbf{F}$ & :the deformation gradient tensor \\
\hline $\mathbf{Z}$ & :the displacement gradient tensor \\
\hline $\mathbf{C}$ & :the right Cauchy-Green tensor \\
\hline $\mathbf{E}$ & :the Green-Lagrange strain tensor \\
\hline$\Pi$ & :the first Piola-Kirchhoff stress tensor \\
\hline $\mathbf{I}$ & :the identity tensor \\
\hline$I_{c}, I I_{c}, I I I_{c}$ & :principal invariants \\
\hline
\end{tabular}

\section{Homogenization Method for Finite Deformation Problem}

\subsection{Problem Statement and Geometric Prospect}

We assume that the material in the $\operatorname{body}(\Omega)$ reveals heterogeneity on a very fine scale and is characterized by the periodic distribution of a basic structural element $\left(Y_{0}\right)$ as shown in Fig. 1. To measure the changes in the spatial domains, we introduce two scales: a macro-scale $X \in \Omega$ and a micro-scale $Y \in Y_{0}$. Thus the actual domain can be regarded as the product space $\left(\Omega \times Y_{0}\right)$. In the subsequent development, the macroscopic quantity corresponding to the microscopic one is expressed with a bar symbol over the microscopic symbol. The following assumptions of homogenization are employed in the formulation of the homogenization method.

- A macrostructure that consists of a periodic microstructure can be considered to be an approximately equivalent homogeneous substance.

- A microstructure is infinitely fine compared with a macrostructure; the variable defined at each point of the macrostructure corresponds to the volume average of the variables in the microstructure.

The deformation of the microstructure is assumed to be linked to the local values of the macro continuum via

$$
\mathbf{y}=\overline{\mathbf{F}} \mathbf{Y}+\mathbf{w},
$$




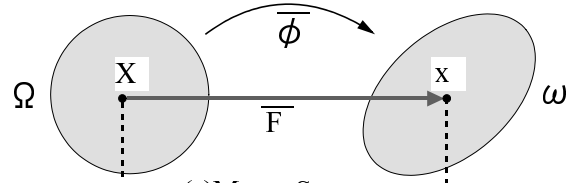

(a)Macro Structure

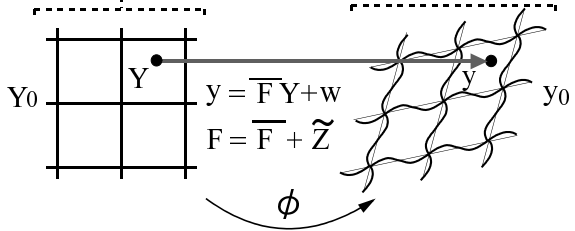

(b)Micro Structure

Fig. 1 Homogenization method for large deformation problems

where $\mathbf{y}$ and $\mathbf{Y}$ are position vectors defined on the microstructure.

The deformation consists of a homogeneous part $\overline{\mathbf{F}} \mathbf{Y}$ and a non-homogeneous superposed field $\mathbf{w}$. Consequently, the following relationships exist between the microscopic and macroscopic deformation gradients.

$$
\begin{aligned}
& \mathbf{F}=\nabla_{Y} \mathbf{y}=\frac{\partial \mathbf{y}}{\partial \mathbf{Y}}=\overline{\mathbf{F}}+\tilde{\mathbf{Z}}, \\
& \overline{\mathbf{F}}=\nabla_{X} \mathbf{x}=\frac{\partial \mathbf{x}}{\partial \mathbf{X}}, \\
& \tilde{\mathbf{Z}}=\nabla_{Y} \mathbf{w}=\frac{\partial \mathbf{w}}{\partial \mathbf{Y}} .
\end{aligned}
$$

Thus increment and variation of the deformation gradients become

$$
\begin{aligned}
& \Delta \mathbf{F}=\Delta \overline{\mathbf{F}}+\Delta \tilde{\mathbf{Z}}=\Delta \overline{\mathbf{F}}+\nabla_{Y} \Delta \mathbf{w}, \\
& \delta \mathbf{F}=\delta \overline{\mathbf{F}}+\delta \tilde{\mathbf{Z}}=\delta \overline{\mathbf{F}}+\nabla_{Y} \delta \mathbf{w} .
\end{aligned}
$$

For the assumptions mentioned above, the macroscopic gradients are related via the volume averages

$$
\overline{\mathbf{F}}=\frac{1}{|V|} \int_{Y_{0}} \mathbf{F} d Y=\frac{1}{|V|} \int_{Y_{0}}(\overline{\mathbf{F}}+\tilde{\mathbf{Z}}) d Y=\overline{\mathbf{F}}+\frac{1}{|V|} \int_{Y_{0}} \tilde{\mathbf{Z}} d Y,
$$

where $V$ is the volume of the microstructure $Y_{0}$. Then, the fluctuation field $\mathbf{w}$ has to satisfy the constraint

$$
\int_{Y_{0}} \tilde{\mathbf{Z}} d Y=\int_{Y_{0}} \frac{\partial \mathbf{w}}{\partial \mathbf{Y}} d Y=\int_{\partial Y_{0}} \mathbf{N} \otimes \mathbf{w} d S=\mathbf{0},
$$

where $\mathbf{N}$ is an outward normal vector on the boundary $\partial Y_{0}$. This constraint is satisfied when $\mathbf{w}$ is periodic.

\subsection{Formulation of Homogenization Method and Finite Element Discretization}

We consider the equilibrium problem with a periodic microstructure. For the homogenization assumptions, the macroscopic virtual work is related via the volume averages and the entire virtual work represents

$$
\int_{\Omega} \overline{\delta \mathbf{F}: \boldsymbol{\Pi}} d X=\int_{\Omega} \frac{1}{|V|} \int_{Y_{0}} \delta \mathbf{F}: \mathbf{\Pi} d Y d X=F_{e x t}(\delta u),
$$

where $F_{\text {ext }}(\delta u)$ is the external virtual work. A similar equation has been reported by Terada et al. ${ }^{(11)}$ using two-scale convergence theory.

By inserting Eq. (6) into Eq. (9), macro and micro equilibrium equations can be derived based on the defined space of the variation.

$$
\bar{G}=\int_{\Omega} \frac{1}{|V|} \int_{Y_{0}} \delta \overline{\mathbf{F}}: \Pi d Y d X=\int_{\Omega} \delta \overline{\mathbf{F}}: \bar{\Pi} d X=F_{\text {ext }}(\delta u)
$$




$$
\begin{aligned}
& G=\int_{Y_{0}} \delta \tilde{\mathbf{Z}}: \boldsymbol{\Pi} d Y=0, \\
& \overline{\mathbf{\Pi}}=\frac{1}{|V|} \int_{Y_{0}} \boldsymbol{\Pi} d Y,
\end{aligned}
$$

which achieves equilibrium under the given boundary condition in the macrostructure and selfequilibrium under the periodic boundary condition Eq. (8) of microscopic displacement in the microstructure. Thus the homogenization method simultaneously satisfies the two equilibrium conditions as described above. To solve the nonlinear equation, the Newton-Raphson method is employed. Then the linearization process provides the following liberalized equations

$$
\begin{aligned}
& \int_{\Omega} \frac{1}{|V|} \int_{Y_{0}} \delta \mathbf{F}: \mathbf{A}: \Delta \mathbf{F} d Y d X=F_{\text {ext }}(\delta u)-\int_{\Omega} \frac{1}{|V|} \int_{Y_{0}} \delta \mathbf{F}: \Pi d Y d X, \\
& \mathbf{A}=\frac{\partial \mathbf{\Pi}}{\partial \mathbf{F}} .
\end{aligned}
$$

Substituting Eqs. (5) and (6) into the above equation yields

$$
\begin{aligned}
& \int_{\Omega} \frac{1}{|V|} \int_{Y_{0}}(\delta \overline{\mathbf{F}}+\delta \tilde{\mathbf{Z}}): \mathbf{A}:(\Delta \overline{\mathbf{F}}+\Delta \tilde{\mathbf{Z}}) d Y d X= \\
& F_{\text {ext }}(\delta u)-\int_{\Omega} \frac{1}{|V|} \int_{Y_{0}}(\delta \overline{\mathbf{F}}+\delta \tilde{\mathbf{Z}}): \Pi d Y d X .
\end{aligned}
$$

By finite element discretization using

$$
\begin{aligned}
& \Delta \overline{\mathbf{F}}=\left[\overline{B^{e}}\right]\left\{\Delta \mathbf{u}^{e}\right\}, \\
& \Delta \tilde{\mathbf{Z}}=\left[B^{e}\right]\left\{\Delta \mathbf{w}^{e}\right\},
\end{aligned}
$$

the left-hand side of Eq. (15) becomes

$$
\begin{aligned}
& \left\{\delta \mathbf{w}^{Q}\right\} \frac{1}{|V|} \int_{Y_{0}}\left[B^{e}\right]^{T}[A]\left[B^{e}\right] d Y\left\{\Delta \mathbf{w}^{Q}\right\}+\left\{\delta \mathbf{w}^{Q}\right\} \frac{1}{|V|} \int_{Y_{0}}\left[B^{e}\right]^{T}[A] d Y\left[\overline{B^{e}}\right]\left\{\Delta \mathbf{u}^{e}\right\}+ \\
& \left\{\delta \mathbf{u}^{e}\right\}\left[\overline{B^{e}}\right]^{T} \frac{1}{|V|} \int_{Y_{0}}[A]\left[B^{e}\right] d Y\left\{\Delta \mathbf{w}^{Q}\right\}+\left\{\delta \mathbf{u}^{e}\right\}\left[\overline{B^{e}}\right]^{T} \frac{1}{|V|} \int_{Y_{0}}[A] d Y\left[\overline{B^{e}}\right]\left\{\Delta \mathbf{u}^{e}\right\} \\
& =\left\{\delta \mathbf{w}^{Q}\right\} \mathbf{K}_{w w}^{Q}\left\{\Delta \mathbf{w}^{Q}\right\}+\left\{\delta \mathbf{w}^{Q}\right\} \mathbf{K}_{w u}^{Q}\left\{\Delta \mathbf{u}^{e}\right\}+\left\{\delta \mathbf{u}^{e}\right\} \mathbf{K}_{u w}^{Q}\left\{\Delta \mathbf{w}^{Q}\right\}+\left\{\delta \mathbf{u}^{e}\right\} \mathbf{K}_{u u}^{Q}\left\{\Delta \mathbf{u}^{e}\right\},
\end{aligned}
$$

while the second term of the right-hand side of Eq. (15) becomes

$$
-\left\{\delta \mathbf{u}^{e}\right\}\left[\bar{B}^{e}\right]^{T} \frac{1}{|V|} \int_{Y_{0}}[\Pi] d Y-\left\{\delta \mathbf{w}^{Q}\right\} \frac{1}{|V|} \int_{Y_{0}}\left[B^{e}\right]^{T}[\Pi] d Y,
$$

at each quadrature point of the macrostructure. The symbol $Q$ denotes the quantity that is evaluated at the macroscopic quadrature point and symbol $e$ the quantity evaluated in the macroscopic element. By assembling these appropriately on the macro continuum, and considering the facultative variations, the following semi-positive definite symmetric matrix is obtained

$$
\left[\begin{array}{ll}
\mathbf{K}_{w w} & \mathbf{K}_{w u} \\
\mathbf{K}_{u w} & \mathbf{K}_{u u}
\end{array}\right]\left\{\begin{array}{c}
\Delta \mathbf{w} \\
\Delta \mathbf{u}
\end{array}\right\}=\left\{\begin{array}{c}
\mathbf{r}_{w} \\
\mathbf{r}_{u}
\end{array}\right\},
$$

where

$$
\begin{aligned}
& \mathbf{K}_{w w}=\int_{\Omega}\left(\frac{1}{|V|} \int_{Y_{0}}\left[B^{e}\right]^{T}[A]\left[B^{e}\right] d Y\right) d X \\
& \mathbf{K}_{w u}=\int_{\Omega}\left(\frac{1}{|V|} \int_{Y_{0}}\left[B^{e}\right]^{T}[A] d Y\right)\left[\overline{B^{e}}\right] d X \\
& \mathbf{K}_{u w}=\int_{\Omega}\left[\overline{B^{e}}\right]^{T}\left(\frac{1}{|V|} \int_{Y_{0}}[A]\left[B^{e}\right] d Y\right) d X \\
& \mathbf{K}_{u u}=\int_{\Omega}\left[\overline{B^{e}}\right]^{T}\left(\frac{1}{|V|} \int_{Y_{0}}[A] d Y\right)\left[\overline{B^{e}}\right] d X \\
& \left\{\mathbf{r}_{w}\right\}=-\int_{\Omega}\left(\frac{1}{|V|} \int_{Y_{0}}\left[B^{e}\right]^{T}[\Pi] d Y\right) d X \\
& \left\{\mathbf{r}_{u}\right\}=\bar{F}_{e x t}-\int_{\Omega}\left[\overline{B^{e}}\right]^{T}\left(\frac{1}{|V|} \int_{Y_{0}}[\Pi] d Y\right) d X .
\end{aligned}
$$


The nonlinear homogenization method solves Eq. (20) for $\Delta \mathbf{u}$ and $\Delta \mathbf{w}$ under the given boundary condition for the macrostructure and the periodic boundary condition (Eq. (8)) for microscopic displacement. The number of degrees of freedom (NDOF) of this matrix is (NDOF of macrostructure + quadrature point of macrostructure $\times$ NDOF of microstructure). An enormous computational cost is also needed for solving the small-scale problem. It is difficult to solve the form given in Eq. (8) due to the limitations of memory, and generally, a transformation into the weak form takes place as described below.

\subsection{Characteristic Deformation}

In a nonlinear problem, to evaluate the response of a microstructure to macroscopic deformation in a similar way to that in a linear problem ${ }^{(10)}$, we obtain the following equation by taking a derivative of Eq. (11) at each quadrature point and substituting Eqs. (5) and (14).

$$
\int_{Y_{0}} \delta \tilde{\mathbf{Z}}: \mathbf{A}: d \tilde{\mathbf{Z}} d Y=-\int_{Y_{0}} \delta \tilde{\mathbf{Z}}: \mathbf{A}: d \overline{\mathbf{F}} d Y
$$

Since the macroscopic deformation gradient is independent of the microscopic integration

$$
\int_{Y_{0}} \delta \tilde{\mathbf{Z}}: \mathbf{A}: \frac{\partial \tilde{\mathbf{Z}}}{\partial \overline{\mathbf{F}}} d Y=-\int_{Y_{0}} \delta \tilde{\mathbf{Z}}: \mathbf{A}: \mathbf{I} d Y
$$

where $\mathbf{I}$ is a fourth order identity tensor and the microstructural response of the macroscopic deformation gradient becomes

$$
\begin{aligned}
& \frac{\partial \tilde{\mathbf{Z}}}{\partial \overline{\mathbf{F}}}=\nabla_{Y}\left(\frac{\partial \mathbf{w}}{\partial \overline{\mathbf{F}}}\right) \equiv-\nabla_{Y} \chi, \\
& \chi \equiv-\frac{\partial \mathbf{w}}{\partial \overline{\mathbf{F}}},
\end{aligned}
$$

where $\chi$ is a third order tensor. This is called the characteristic deformation. The equation above can be substituted into Eq. (28) yielding

$$
\int_{Y_{0}} \delta \tilde{\mathbf{Z}}: \mathbf{A}: \nabla_{Y} \chi d Y=\int_{Y_{0}} \delta \tilde{\mathbf{Z}}: \mathbf{A}: \mathbf{I} d Y .
$$

By finite element discretization, the gradient of $\chi$ becomes

$$
\nabla_{Y \chi}=\left[B^{e}\right]\left[\chi^{e}\right]
$$

in a microscopic finite element. $\left[\chi^{e}\right]$ is the derivative of the microscopic displacement for each component of $\overline{\mathbf{F}}$; in other words, it is the matrix given below with nine kinds of characteristic deformation.

$$
\left[\chi^{e}\right]=\left[\begin{array}{cccc}
\chi_{111}^{e} & \chi_{112}^{e} & \cdots & \chi_{133}^{e} \\
\chi_{211}^{e} & \chi_{212}^{e} & \cdots & \chi_{233}^{e} \\
\vdots & \vdots & \ddots & \vdots \\
\chi_{n 11}^{e} & \chi_{n 12}^{e} & \cdots & \chi_{n 33}^{e}
\end{array}\right]
$$

where $\mathrm{n}$ is the NDOF of one finite element of the microstructure. The matrix equation becomes

$$
\begin{aligned}
& \mathbf{K}_{\chi}\left[\chi^{Q}\right]=\left[\mathbf{r}_{\chi}\right], \\
& \mathbf{K}_{\chi}=\int_{Y_{0}}\left[B^{e}\right]^{T}[A]\left[B^{e}\right] d Y, \\
& {\left[\mathbf{r}_{\chi}\right]=\int_{Y_{0}}\left[B^{e}\right]^{T}[A][I] d Y,}
\end{aligned}
$$

from Eq. (31) about one microstructure. $\left[\chi^{Q}\right]$ denotes that the quantity is evaluated at the macroscopic quadrature point. The value of $\left[\chi^{Q}\right]$ which is a (NDOF of the microstructure) row $\times 9$ column matrix can be obtained by assembling $\left[\chi^{e}\right] .[I]$ is a ninth order identity matrix, consisting of nine column vectors $\left\{I_{i}\right\}(i=1 \ldots 9)$. Thus nine solutions can be obtained for the right-hand side of Eq. (36), one for each $\left\{I_{i}\right\}$ corresponding to a component of the deformation gradient. $\left[\chi^{Q}\right]$ can then be obtained by solving each different version of the right-hand side of the above equation. 


$$
\frac{1}{|V|} \mathbf{K}_{\chi}\left[\chi^{Q}\right]\left[\overline{B^{e}}\right]=\frac{1}{|V|}\left[\mathbf{r}_{\chi}\right]\left[\overline{B^{e}}\right]
$$

Now, by using Eq. (18)

$$
\begin{aligned}
& \frac{1}{|V|} \mathbf{K}_{\chi}=\mathbf{K}_{w w}^{Q}, \\
& \frac{1}{|V|}\left[\mathbf{r}_{\chi}\right]\left[\overline{B^{e}}\right]=\mathbf{K}_{w u}^{Q},
\end{aligned}
$$

thus

$$
\mathbf{K}_{w w}^{Q}\left[\chi^{Q}\right]\left[\overline{B^{e}}\right]=\mathbf{K}_{w u}^{Q} .
$$

The total is taken at all macroscopic quadrature points in respect of the above equation

$$
\begin{aligned}
& \mathbf{K}_{w w}[\chi][\bar{B}]=\mathbf{K}_{w u}, \\
& {[\bar{B}] \equiv \sum_{i}\left[\overline{B^{e}}\right],} \\
& {[\chi] \equiv \sum_{i}\left[\chi^{Q}\right]}
\end{aligned}
$$

where $[\chi]$ is a matrix consisting of (quadrature points of macrostructure $\times$ NDOF of microstructure) rows $\times 9$ columns.

The characteristic deformations are the deformation increments for unit macroscopic deformation gradients at a particular moment and these describe the material properties and strain distribution of the microstructure. Equation (27) can also be considered a linear approximation of the microscopic deformation. Therefore, the update of the microscopic deformation by

$$
\Delta \mathbf{w}=-\chi: \Delta \overline{\mathbf{F}}
$$

corresponds to the Forward Euler method for microscopic deformation from Eq. (30).

\subsection{Homogenization Method using Characteristic Deformation Mode Superposition}

In the mode superposition homogenization method, the microscopic displacement increment is approximately obtained by the linear combination of the previously calculated characteristic deformation $0 \chi$ and the scaling factor in Eq. (44) as ${ }^{(6)}$

$$
\Delta w_{k} \doteqdot-{ }_{0} \chi_{k p Q} \Delta \alpha_{p Q},
$$

where $\alpha$ is the scaling factor for each mode. Inserting Eq. (45) into Eq. (20) yields the matrix

$$
\begin{gathered}
{\left[\begin{array}{cc}
{[0 \chi]^{T}} & \mathbf{0} \\
\mathbf{0} & \mathbf{I}
\end{array}\right]\left[\begin{array}{cc}
\mathbf{K}_{w w} & \mathbf{K}_{w u} \\
\mathbf{K}_{u w} & \mathbf{K}_{u u}
\end{array}\right]\left[\begin{array}{cc}
{[0 \chi]} & \mathbf{0} \\
\mathbf{0} & \mathbf{I}
\end{array}\right]\left\{\begin{array}{c}
\Delta \alpha \\
\Delta \mathbf{u}
\end{array}\right\}=\left[\begin{array}{cc}
{[0 \chi]^{T}} & \mathbf{0} \\
\mathbf{0} & \mathbf{I}
\end{array}\right]\left\{\begin{array}{c}
\mathbf{r}_{w} \\
\mathbf{r}_{u}
\end{array}\right\}} \\
{\left[\begin{array}{cc}
{[0 \chi]^{T} \mathbf{K}_{w w}[0 \chi]} & {[0 \chi]^{T} \mathbf{K}_{w u}} \\
\mathbf{K}_{u w}[0 \chi & \mathbf{K}_{u u}
\end{array}\right]\left\{\begin{array}{c}
\Delta \alpha \\
\Delta \mathbf{u}
\end{array}\right\}=\left\{\begin{array}{c}
{[0 \chi]^{T} \mathbf{r}_{w}} \\
\mathbf{r}_{u}
\end{array}\right\} .}
\end{gathered}
$$

where $[0 \chi]$ is the same kind of matrix as $[\chi]$. The above equation can be represented as

$$
\left[\begin{array}{ll}
\mathbf{K}_{\alpha \alpha} & \mathbf{K}_{\alpha u} \\
\mathbf{K}_{u \alpha} & \mathbf{K}_{u u}
\end{array}\right]\left\{\begin{array}{c}
\Delta \alpha \\
\Delta \mathbf{u}
\end{array}\right\}=\left\{\begin{array}{c}
\mathbf{r}_{\alpha} \\
\mathbf{r}_{u}
\end{array}\right\},
$$

where,

$$
\begin{aligned}
& \mathbf{K}_{\alpha \alpha}=\int_{\Omega}\left(\frac{1}{|V|} \int_{Y_{0}}\left[0 \chi^{e}\right]^{T}\left[B^{e}\right]^{T}[A]\left[B^{e}\right]\left[0 \chi^{e}\right] d Y\right) d X \\
& \mathbf{K}_{\alpha u}=-\int_{\Omega}\left(\frac{1}{|V|} \int_{Y_{0}}\left[0 \chi^{e}\right]^{T}\left[B^{e}\right]^{T}[A] d Y\right)\left[\overline{B^{e}}\right] d X
\end{aligned}
$$




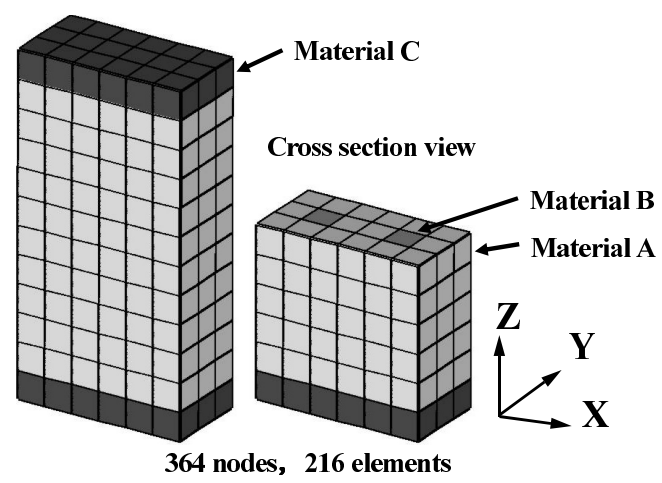

Fig. 2 Simplified cell model

Table. 1 Material constants for simplified cell model

\begin{tabular}{|c|c|c|}
\hline & $E[\mathrm{kPa}]$ & $v$ \\
\hline Material A in Fig.2 & 0.1 & 0.3 \\
\hline Material B in Fig.2 & 60.0 & 0.3 \\
\hline Material C in Fig.2 & 100.0 & 0.3 \\
\hline
\end{tabular}

$$
\begin{aligned}
\mathbf{K}_{u \alpha} & =-\int_{\Omega}\left[\overline{B^{e}}\right]^{T}\left(\frac{1}{|V|} \int_{Y_{0}}[A]\left[B^{e}\right]\left[0 \chi^{e}\right] d Y\right) d X \\
\mathbf{K}_{u u} & =\int_{\Omega}\left[\overline{B^{e}}\right]^{T}\left(\frac{1}{|V|} \int_{Y_{0}}[A] d Y\right)\left[\overline{B^{e}}\right] d X \\
\left\{\mathbf{r}_{u}\right\} & =\bar{F}_{e x t}-\int_{\Omega}\left[\overline{B^{e}}\right]^{T}\left(\frac{1}{|V|} \int_{Y_{0}}[\Pi] d Y\right) d X \\
\left\{\mathbf{r}_{\alpha}\right\} & =\int_{\Omega}\left(\frac{1}{|V|} \int_{Y_{0}}\left[0 \chi^{e}\right]^{T}\left[B^{e}\right]^{T}[\Pi] d Y\right) d X .
\end{aligned}
$$

We obtain the matrix with unknowns $\Delta \alpha$ and $\Delta \mathbf{u}$. Because the NDOF of the matrix is reduced to $(\mathrm{NDOF}$ of macrostructure + quadrature point of macrostructure $\times 9$ ), significant computational cost is saved.

This technique is however, an approximate means of achieving equilibrium in a range of displacements representing linear combinations of $\chi_{0}$, as it is clear from Eq. (54) that $\chi_{0}$ has an effect on equilibrium. In this way, to approximate the deformation in limited deformation patterns, an approximation error is created depending on the analysis case. This is illustrated using the simplified cell model shown in Fig. 2. For the constitutive equation, the St. Venant hyperelastic material is adopted and the material constants are given in Table 1. The macrostructure consists of one element. The deformation of mode superposition method was compared with the exact method under the same load boundary condition. The initial characteristic deformation used for $\chi_{0}$ is shown in Fig. 4, while examples of deformation of the microstructure are also shown in Fig. 3. Smooth deformation is achieved for shear deformation in the exact method, while irregular deformation is generated by the mode superposition method.

Such a difference has an effect on the convergence solution of the macroscopic deformation and stress through the framework of homogenization. On the other hand, tensile deformation can be confirmed to be approximately correct. The mode superposition method has low computational cost, but it has an approximation error, although this agrees globally with the exact method.

\section{Algorithm for Nonlinear Homogenization Method}

\subsection{Generalized Algorithm}

In Eq. (20), $\{\Delta w\}$ can be statically condensed at the element quadrature point level. $\{\Delta w\}$ becomes

$$
\{\Delta \mathbf{w}\}=\mathbf{K}_{w w}^{-1}\left(\left\{\mathbf{r}_{w}\right\}-\mathbf{K}_{w u}\{\Delta \mathbf{u}\}\right) .
$$




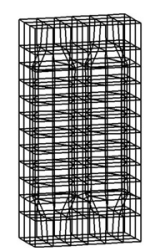

$\mathrm{XX}$

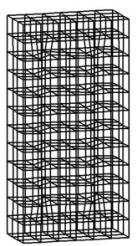

YX

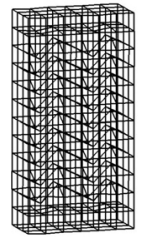

ZX

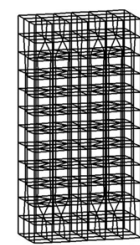

$\mathrm{XY}$

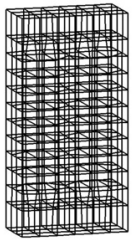

YY

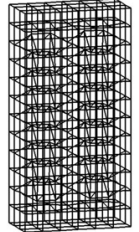

$\mathrm{ZY}$

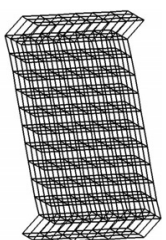

$\mathrm{XZ}$

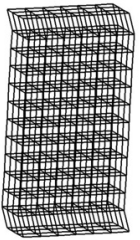

$\mathrm{YZ}$

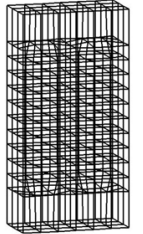

$\mathrm{ZZ}$

Fig. 3 Characteristic deformations in the initial state

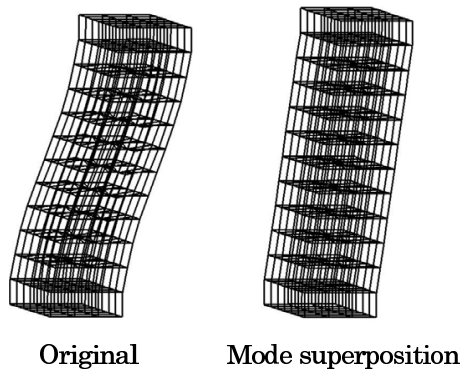

(a) Shear
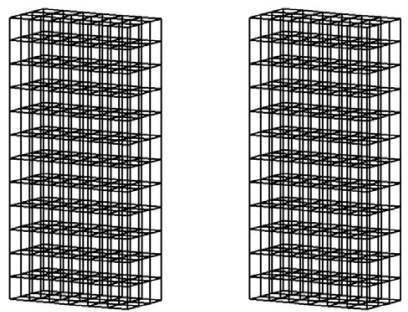

Original

Mode superposition

(b) Tension

Fig. 4 Disadvantage of characteristic deformation mode superposition 
$\{\Delta w\}$ vanishes when the above equation is substituted in the macroscopic equilibrium equation

$$
\left(\mathbf{K}_{u u}-\mathbf{K}_{u w} \mathbf{K}_{w w}^{-1} \mathbf{K}_{w u}\right)\{\Delta \mathbf{u}\}=\left\{\mathbf{r}_{u}\right\}-\mathbf{K}_{u w} \mathbf{K}_{w w}^{-1}\left\{\mathbf{r}_{w}\right\} .
$$

Now, the microscopic equilibrium hypothesized for $\overline{\mathbf{F}}$ at this time is

$$
\left\{\mathbf{r}_{w}\right\}=0 .
$$

By using Eqs. (41) and (55), we obtain ${ }^{(1)}$

$$
\{\Delta \mathbf{w}\}=-\mathbf{K}_{w w}^{-1} \mathbf{K}_{w u}\{\Delta \mathbf{u}\}=-[\chi][\overline{\mathbf{B}}]\{\Delta \mathbf{u}\} .
$$

By using Eqs. (41), (55), and (56) we can represent

$$
\left(\mathbf{K}_{u u}-\mathbf{K}_{u w}[\chi][\overline{\mathbf{B}}]\right)\{\Delta \mathbf{u}\}=\left\{\mathbf{r}_{u}\right\},
$$

where $\left(\mathbf{K}_{u u}-\mathbf{K}_{u w}[\chi][\overline{\mathbf{B}}]\right)$ is called the homogenized tangent stiffness.

In differential equation form, from Eqs. (11), (15), and (29), this becomes

$$
\begin{aligned}
& \int_{\Omega} \delta \overline{\mathbf{F}}:\left(\frac{1}{|V|} \int_{Y_{0}}\left(\mathbf{A}:\left(\mathbf{I}-\nabla_{Y} \chi\right)\right) d Y\right): \Delta \overline{\mathbf{F}} d X \\
& =\overline{\mathbf{F}}_{\text {ext }}-\int_{\Omega} \delta \overline{\mathbf{F}}:\left(\frac{1}{|V|} \int_{Y_{0}} \boldsymbol{\Pi} d Y\right) d X .
\end{aligned}
$$

Microscopic equilibrium Eq. (57)

$$
r_{w}=\int_{Y_{0}} \delta \tilde{\mathbf{Z}}: \boldsymbol{\Pi} d Y=\mathbf{0},
$$

is a prerequisite of the above equation. To satisfy this nonlinear prerequisite, a NewtonRaphson iteration is needed. Thus the above equation is linearized with respect to $\mathbf{w}$ while $\overline{\mathbf{F}}$ is fixed, and then discretized by the finite element method using Eq. (17). At each quadrature point, the linearized self-equilibrated equation becomes

$$
\int_{Y_{0}}\left[B^{e}\right]^{T}[A]\left[B^{e}\right] d Y\left\{\Delta \mathbf{w}^{Q}\right\}=-\int_{Y_{0}}\left[B^{e}\right]^{T}[\Pi] d Y .
$$

In the generalized algorithm, three kinds of calculation have to be dealt with in each iteration.

( 1 ) Update macroscopic tangential homogenization $\chi$ using Eq. (34).

( 2 ) Solve microscopic equilibrium problem Eq. (61) and obtain the convergence solution $\{\mathbf{w}\}$ while $\overline{\mathbf{F}}$ is fixed.

(3) Solve the linearized macroscopic equilibrium equation Eq. (59) to obtain $\{\mathbf{u}\}$.

Processes 1 and 2 need to be solved at all quadrature points of the macrostructure and it is known that this takes up the greatest part of the calculation load ${ }^{(2)}$. These processes require prohibitive computational cost and actual numerical simulation is difficult.

\subsection{Block LU Factorization Algorithm}

We now present the algorithm that decreases the residual of each scale simultaneously using the block LU factorization algorithm without microscopic convergence in the macroscopic iteration as in the conventional algorithm. By Block LU factorization Eq. (20) becomes

$$
\left[\begin{array}{cc}
\mathbf{K}_{w w} & \mathbf{0} \\
\mathbf{K}_{u w} & \mathbf{S}
\end{array}\right]\left[\begin{array}{cc}
\mathbf{I} & \mathbf{K}_{w w}^{-1} \mathbf{K}_{w u} \\
\mathbf{0} & \mathbf{I}
\end{array}\right]\left\{\begin{array}{c}
\Delta \mathbf{w} \\
\Delta \mathbf{u}
\end{array}\right\}=\left\{\begin{array}{l}
\mathbf{r}_{w} \\
\mathbf{r}_{u}
\end{array}\right\},
$$

where $\mathbf{S}$ is called the Schur-Complement. Referring to Eq. (41), $\mathbf{S}$ can represent

$$
\mathbf{S}=\mathbf{K}_{u u}-\mathbf{K}_{u w} \mathbf{K}_{w w}^{-1} \mathbf{K}_{w u}=\mathbf{K}_{u u}-\mathbf{K}_{u w}[\chi][\overline{\mathbf{B}}] .
$$

which corresponds to the homogenized tangent stiffness of Eq. (59). The solution process for Eq. (63) is composed of the forward substitution

$$
\left[\begin{array}{ll}
\mathbf{K}_{w w} & \mathbf{0} \\
\mathbf{K}_{u w} & \mathbf{S}
\end{array}\right]\left\{\begin{array}{c}
\Delta \tilde{\mathbf{w}} \\
\Delta \tilde{\mathbf{u}}
\end{array}\right\}=\left\{\begin{array}{l}
\mathbf{r}_{w} \\
\mathbf{r}_{u}
\end{array}\right\},
$$


and the backward substitution

$$
\left\{\begin{array}{c}
\Delta \tilde{\mathbf{w}} \\
\Delta \tilde{\mathbf{u}}
\end{array}\right\}=\left[\begin{array}{cc}
\mathbf{I} & \mathbf{K}_{w w}^{-1} \mathbf{K}_{w u} \\
\mathbf{0} & \mathbf{I}
\end{array}\right]\left\{\begin{array}{c}
\Delta \mathbf{w} \\
\Delta \mathbf{u}
\end{array}\right\}
$$

Then, from Eqs. (41), (65), and (66)

$$
\begin{aligned}
& \mathbf{K}_{w w}\{\Delta \tilde{\mathbf{w}}\}=\left\{\mathbf{r}_{w}\right\}, \\
& \mathbf{S}\{\Delta \mathbf{u}\}=\left\{\mathbf{r}_{u}\right\}-\mathbf{K}_{u w}\{\Delta \tilde{\mathbf{w}}\}, \\
& \{\Delta \mathbf{w}\}=\{\Delta \tilde{\mathbf{w}}\}-\mathbf{K}_{w w}^{-1} \mathbf{K}_{w u}\{\Delta \mathbf{u}\}=\{\Delta \tilde{\mathbf{w}}\}-[\chi] \Delta \overline{\mathbf{F}},
\end{aligned}
$$

hold. The calculation procedure is described below.

( 1 ) Solve linearized microscopic equilibrium equation Eq. (67) to obtain $\{\Delta \mathbf{w}\}$.

( 2 ) Solve linearized microscopic equilibrium equation Eq. (68) to obtain $\{\Delta \mathbf{u}\}$. In addition, this equation is equivalent to Eq. (56).

( 3 ) Update $\{\Delta \mathbf{w}\}$ using Eq. (69). We have already obtained $[\chi]$ when calculating $\mathbf{S}$.

Three kinds of matrix, namely micro, macro, and Schur-Complement, need to be solved in each iteration. The computational cost of this algorithm is lower than the generalized algorithm, but the Schur-Complement update is still expensive as described above. A similar algorithm for a differential equation using the Block-Newton method has been proposed by Yamada et al. ${ }^{(12)}$.

\subsection{Mode Superposition as an Approximation of the Macroscopic Tangent Stiffness}

In the block LU factorization algorithm, most of the computational cost is consumed by updating the Schur-Complement. But the Schur-Complement is part of the macroscopic tangent stiffness as described above and as such does not influence the equilibrium directly. To avoid updating the Schur-Complement, we propose using Mode superposition as an approximation of the Schur-Complement. The solution agrees completely with the exact method by making a convergence judgment for the microscopic and macroscopic residuals. The calculation procedure is described below.

( 1 ) Solve the linearized microscopic equilibrium equation Eq. (67) to obtain $\{\Delta \tilde{\mathbf{w}}\}$.

( 2 ) From Eq. (48), solve the mode superposition-based linearized multi-scale equation

$$
\left[\begin{array}{ll}
\mathbf{K}_{\alpha \alpha} & \mathbf{K}_{\alpha u} \\
\mathbf{K}_{u \alpha} & \mathbf{K}_{u u}
\end{array}\right]\left\{\begin{array}{l}
\Delta \alpha \\
\Delta \mathbf{u}
\end{array}\right\}=\left\{\begin{array}{l}
\mathbf{r}_{\alpha} \\
\mathbf{r}_{u}
\end{array}\right\}
$$

to obtain $\{\Delta \mathbf{u}\}$. Then, $\mathbf{S}$ is approximated by the range of the mode superposition method and the update of $\mathbf{S}$ can be omitted.

( 3 ) The assumption of the mode superposition method follows from Eqs. (44), (45), and (69), and $\{\Delta \mathbf{w}\}$ is updated using

$$
\{\Delta \mathbf{w}\}=\{\Delta \tilde{\mathbf{w}}\}-[0 \chi]\{\Delta \alpha\} .
$$

The meaning of the algorithm is now given. Block UL factorization is applied to Eq. (70).

$$
\begin{aligned}
& \mathbf{K}_{\alpha \alpha}\{\Delta \tilde{\alpha}\}=\left\{\mathbf{r}_{\alpha}\right\}, \\
& \left(\mathbf{K}_{u u}-\mathbf{K}_{u \alpha} \mathbf{K}_{\alpha \alpha}^{-1} \mathbf{K}_{\alpha u}\right)\{\Delta \mathbf{u}\}=\left\{\mathbf{r}_{u}\right\}-\mathbf{K}_{u \alpha}\{\Delta \tilde{\alpha}\}, \\
& \{\Delta \alpha\}=\{\Delta \tilde{\alpha}\}-\mathbf{K}_{\alpha \alpha}^{-1} \mathbf{K}_{\alpha u}\{\Delta \mathbf{u}\} .
\end{aligned}
$$

In Eq. (73), the macroscopic displacement is updated using

$$
\left(\mathbf{K}_{u u}-\mathbf{K}_{u \alpha} \mathbf{K}_{\alpha \alpha}^{-1} \mathbf{K}_{\alpha u}\right),
$$

which can be considered an approximation of the homogenized tangent stiffness. Then, using Eqs. (41) and (64), the Schur-Complement becomes

$$
\begin{aligned}
& \mathbf{K}_{u w} \mathbf{K}_{w w}^{-1} \mathbf{K}_{w u}=\mathbf{K}_{u w}[\chi] \overline{\mathbf{B}} \\
& =\mathbf{K}_{u w}[\chi]\left([\chi]^{T} \mathbf{K}_{w w}[\chi]\right)^{-1}\left([\chi]^{T} \mathbf{K}_{w w}[\chi]\right) \overline{\mathbf{B}} \\
& =\mathbf{K}_{u w}[\chi]\left([\chi]^{T} \mathbf{K}_{w w}[\chi]\right)^{-1}[\chi]^{T} \mathbf{K}_{w u}
\end{aligned}
$$




$$
\begin{aligned}
& \doteqdot \mathbf{K}_{u w}[0 \chi]\left([0 \chi]^{T} \mathbf{K}_{w w}[0 \chi]\right)^{-1}[0 \chi]^{T} \mathbf{K}_{w u} \\
& =\mathbf{K}_{u \alpha} \mathbf{K}_{\alpha \alpha}^{-1} \mathbf{K}_{\alpha u} .
\end{aligned}
$$

In the mode superposition method, since the homogenized tangent stiffness corresponds to the exact value with the mode updated at every Newton-Raphson iteration as described above, this method can give an approximation mean of the homogenized tangent stiffness by decreasing the number of times that the mode is updated.

In the homogenization method, the micro and macro equilibrium conditions must be achieved simultaneously. To evaluate the residual in each scale, micro and macro equations need to be solved in each iteration and this defines the minimum computational cost. Hence, a requirement for low computational cost in nonlinear homogenization algorithms is to approximate the Schur-Complement effectively and accurately.

\section{Numerical Example}

\subsection{Comparison of Computational Cost and Convergence}

We compare computational cost and convergence in the three methods, namely the Generalized algorithm (GA), Block LU factorization algorithm (BFA) and the algorithm using mode superposition (MSA). We did a 20\% tensile test using the mesh of the block as the microstructure as shown in Fig. 5, and the mesh of one element of an 8-node element as macrostructure. The minimum size of the mesh is constructed from $27(3 \times 3 \times 3)$ elements as shown in Fig. 5(a) and the stiffness of the center element is different.

The NDOF is adjusted by adding the same number of minimum units in each direction (e.g. Fig. 5(b)). The St. Venant hyperelastic material is used for the constitutive equation

$$
\begin{aligned}
& W=\frac{1}{2} \lambda(t r \mathbf{E})^{2}+\mu \mathbf{E}: \mathbf{E}, \\
& \mathbf{S}=\frac{\partial W}{\partial \mathbf{E}}=(\lambda \mathbf{I} \otimes \mathbf{I}+2 \mu \mathbf{l}): \mathbf{E}=\mathbf{C}: \mathbf{E}, \\
& l_{i j k l}=\delta_{i k} \delta_{j l}, \\
& \lambda=\frac{E v}{(1+v)(1-2 v)}, \quad \mu=G=\frac{E}{2(1+v)},
\end{aligned}
$$

where $\mathbf{I}$ is a second order identity tensor, $\lambda$ and $\mu$ are lame constants, and $E$ and $v$ are, respectively, Young's modulus and Poisson's ratio. $\mathbf{C}$ is a fourth order constant elasticity tensor and the relationship between $\boldsymbol{S}$ and $\boldsymbol{E}$ is linear. The material constants are shown in Table 2.

The relationship between calculation time and NDOF in each algorithm is depicted in Fig. 6, while the breakdown of calculation time and number of iterations in each process are given in Table 3, where the NDOF is 46875 . Convergence is judged to occur when the root sum square is $1 \times 10^{-10}$ times the initial value. In the BFA, calculation time is reduced only $20 \%$ compared with the GA. Because the microscopic and macroscopic problems are solved individually in the GA, the Newton-Raphson methods converged after about 4 iterations. On the other hand, the BFA deals with each system separately and 9 iterations were needed. It can be confirmed that the reason for only a $20 \%$ reduction according to Table 3 , is as a result of the increase in computational cost due to updating the Schur-Complement. In this analysis, because an iterative solver (GMRES method) is used for the large-scale calculation, it has to solve 9 different right-hand sides to update the Schur-Complement. If a direct method were used, because the result of the LU decomposition of the stiffness matrix can be reused for the different right-hand side solutions and it only solves the equation a single time in practice, the computational cost of the BFA would show a substantially greater decrease than the GA.

We confirmed that the computational cost of the MSA is far less than the other algorithms. The iterations were terminated at the same level as in the BFA but the homogenized tangent stiffness was approximated. Since the microscopic equation is approximately solved in Eqs. (70) and (71) with Eq. (67) by the MSA, the microscopic equation is effectively solved twice. This accelerates convergence. Although we adopted the St. Venant hyperelastic 


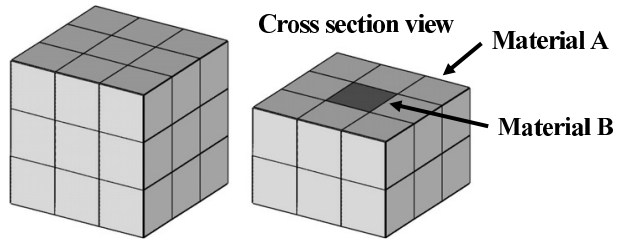

(a) 64 nodes, 27 elements (Minimum size)

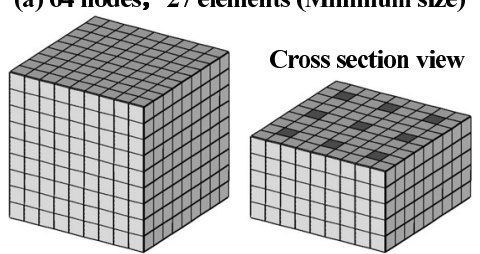

(b)1000 nodes, 729 elements

Fig. 5 FE models for the calculation time evaluation

Table. 2 Material constants for the models in the calculation time evaluation

\begin{tabular}{|c|c|c|}
\hline & $E[\mathrm{kPa}]$ & $v$ \\
\hline Material A in Fig.5 & 0.1 & 0.3 \\
\hline Material B in Fig.5 & 10.0 & 0.3 \\
\hline
\end{tabular}

material with relatively weak nonlinearity, iterations did not increase in the large deformation although initial characteristic deformation was used for $0 \chi$. However, a periodic update of $0 \chi$ is required in the case of a strong nonlinear material such as an elasto-plastic material. If $0 \chi$ is updated in every iteration, the homogenized tangent stiffness corresponds to the exact value as described in Eq. (76).

\subsection{Application to ventricle-cardiomyocyte analysis}

We applied the MSA to a simplified left ventricle-cardiomyocyte model as shown in Figs. 7 and 8 with the NDOF of the microstructure being 9657 . The St. Venant hyperelastic material is adopted for the constitutive equation and the material constants are given in Table 4. The left ventricle model is based on MR imaging, with the ventricle wall divided into three layers, i.e., endocardial, middle and epicardial layers, with fiber directions of $-60,0$, and 60 degrees, respectively, relative to the plain perpendicular to the long axis of the $\mathrm{LV}$. The fiber direction in the cardiomyocyte model, shown in Fig. 8, is in the y-direction. The usual St. Venant hyperelastic material is assumed for the base and apex, while a homogenization method is applied to the middle part of 792 elements. Since there are 8 quadrature points at each element, the total number in the cardiomyocyte model is 6336 . We use a PC-cluster composed of 99 Pentium $4(3.2 \mathrm{GHz})$ PCs connected to each other via Myrinet. Considering the size of the problem, convergence is deemed to occur when the root sum square of the macro and micro residuals is $1 \times 10^{-5}$ times the initial value. The fiber direction is taken into consideration by multiplying the rotational tensor when the micro/macro tensors are exchanged. Since the intracellular matrix is a function of the parameter that represents cardiomyocyte excitation, this

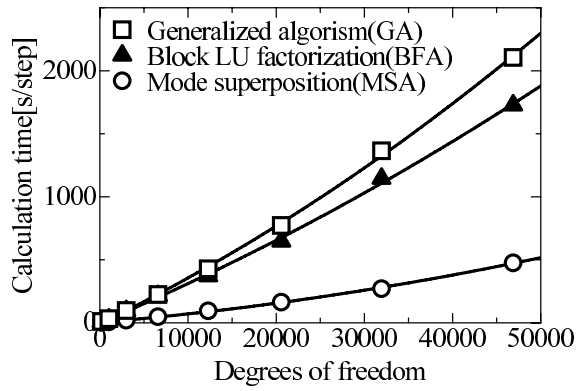

Fig. 6 Comparison of calculation time 
Table. 3 Calculation time and number of iterations in each process

\begin{tabular}{|c|c|c|c|c|}
\hline & Calculation of $\chi[\mathrm{s}]$ & $\begin{array}{c}\text { Calculation of equilibrium in } \\
\text { the microscopic model [s] }\end{array}$ & Total [s] & Iteration \\
\hline GA & 746 & 1296 & 2105 & 5 \\
\hline BFA & 1251 & 372 & 1727 & 9 \\
\hline MSA & 16 & 300 & 475 & 8 \\
\hline
\end{tabular}

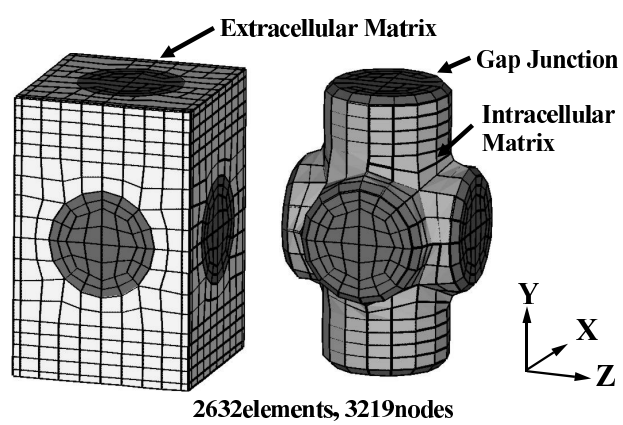

Fig. 7 FE meshes of microscopic model

parameter is adjusted at every time step to represent the contraction force. The deformation of the ventricle and microstructure in the diastole is shown in Fig. 9. The calculation time is 114 [s/iteration], $456[\mathrm{~s} / \mathrm{step}$ ] and the number of iterations is about 4 for each step. In this analysis, dilation of the left ventricle by pressure and excitation contraction is generated as a result of the 6336 cardiomyocyte model, which shows the different electrical and mechanical behavior. The proposed methods thus enable us to deal with large scale problems.

Table. 4 Material constants for the cell model

\begin{tabular}{|c|c|c|}
\hline & $E[\mathrm{kPa}]$ & $v$ \\
\hline Gap Junction & 10.0 & 0.3 \\
\hline Intracellular Matrix & 5.0 & 0.3 \\
\hline Extracellular Matrix & 0.1 & 0.3 \\
\hline
\end{tabular}

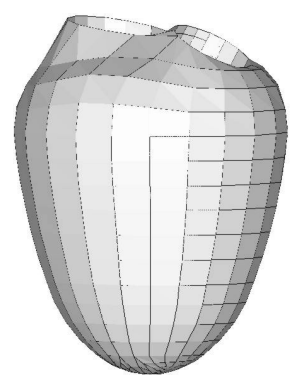

(a) FE mesh

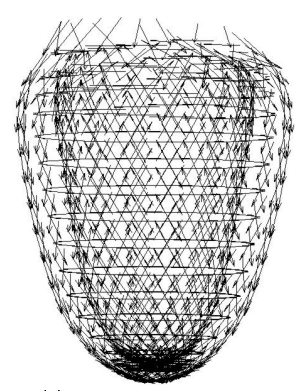

(b) Fiber orientation ents, 8 quadrature points

Fig. 8 FE meshes of macroscopic model

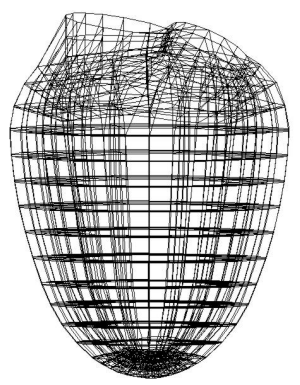

(a) Macroscopic structure

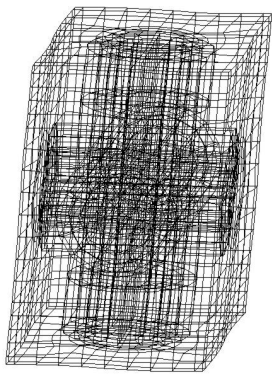

(b) Microscopic structure

Fig. 9 Deformations in diastole 


\section{Conclusion}

To reduce the computational cost of the nonlinear homogenization method, the two algorithms listed below were proposed.

(1) An algorithm which decreases the residual of each scale simultaneously using the block LU factorization.

(2) An algorithm which approximates the homogenization tangent stiffness using the mode superposition method to omit updating the Schur-Complement.

Both the Block LU factorization algorithm and the algorithm using mode superposition can drastically reduce the computational cost compared with the conventional method. Finally, the algorithm using mode superposition applied to the left ventricle of the heart with parallel computation was presented as an example of a large scale calculation.

\section{Acknowledgment}

This work was supported by Core Research for Evolutional Science and Technology, Japan Science and Technology Agency.

\section{References}

( 1 ) Terada, K. and Kikuchi, N., A class of general algorithms for multi-scale analyses of heterogeneous media, Computer Methods in Applied Mechanics and Engineering, 190, No. 40-41(2001), pp.5427-5464.

( 2 ) Matsui, K., Terada, K. and Yuge, K., Two-scale finite element analysis of heterogeneous solids with periodic microstructures, Computers $\mathcal{E}$ Structures, 82(2004) pp.593-606.

( 3 ) Takano, N., Ohnishi, Y., Zako, M. and Nishiyabu, K., Microstructure-based Deepdrawing Simulation of Knitted Fabric Reinforced Thermoplastics by Homogenization Theory, International Journal of Solids and Structures, 38(2001), pp.6333-6356.

( 4 ) Rohan, E., Sensitivity strategies in modelling heterogeneous media undergoing finite deformation, Mathematics and Computers in Simulation, 61(2003), pp.261-270.

( 5 ) Moulinec, H. and Suquet, P., A numerical method for computing the overall response of nonlinear composites with complex microstructure, Computer Methods in Applied Mechanics and Engineering, 157(1998), pp.69-94.

( 6 ) Yamamoto, M., Hisada, T. and Noguchi, H., Homogenization Method for Large Deformation Problem by Using Characteristic Deformation Mode Superposition (in Japanese), Trans. Jpn. Soc. Mech. Eng., 67(2001), pp.1877-1884.

( 7 ) Okada, J. and Hisada, T., Homogenization Method for Mixed Finite Element Analysis of Incompressible Hyper-Elastic Materials (in Japanese), Proceedings of the Conference on Computational Engineering and Science, 11(2006), pp.79-82.

( 8 ) Okada, J. and Hisada, T., Homogenization Method for Mixed Finite Element Analysis of Incompressible Viscoelastic Materials (in Japanese), Proceedings of the 19th Computational Mechanics Conference, No.06-9(2006), pp.45-46.

( 9 ) Miehe, C., Schroder, J. and Schotte, J., Computational Homogenization Analysis in Finite Plasticity. Simulation of Texture Development in Polycrystalline Materials, Computer Methods in Applied Mechanics and Engineering, 171(1999), pp.387-418.

(10) Terada, K. and Kikuchi, N., Introduction to the method of homogenization (in Japanese), Maruzen, 2003.

(11) Terada, K., Saiki, I., Matsui, K. and Yamakawa, Y., Two-scale kinematics and linearization for simultaneous two-scale analysis of periodic heterogeneous solids at finite strain, Computer Methods in Applied Mechanics and Engineering, 192(2003), pp.3531-3563.

(12) Yamada, T. and Matsui, K., Numerical Procedure for Nonlinear Multiscale Problems Based on Block Newton Method (in Japanese), Proceedings of the Conference on Computational Engineering and Science, 12(2007), pp.309-312. 